\title{
Medical Outcomes Study Social Activities
}

National Cancer Institute

\section{Source}

National Cancer Institute. Medical Outcomes Study Social Activities. NCI Thesaurus. Code C110968.

A survey of multiple dimensions of quality of life, representing the social activities scale. 